\title{
Use of QT intervals for a more accurate diagnose of syncope and evaluation of syncope severity
}

\author{
C. Buttà, ${ }^{1}$ A. Tuttolomondo, ${ }^{2}$ A. Casuccio, ${ }^{3}$ D. Di Raimondo, ${ }^{1}$ L. Giarrusso, ${ }^{1}$ G. Micelii, ${ }^{1}$ \\ S. Lo Vecchio, ${ }^{1}$ B. Canino, ${ }^{4}$ G. Licata, ${ }^{2}$ A. Pinto ${ }^{1}$
}

${ }^{1}$ U.O.C. Medicina Vascolare, Dipartimento Biomedico di Medicina Interna e Specialistica, Università degli Studi di, Palermo, Italy

${ }^{2}$ U.O.C. Medicina Interna e Cardioangiologia, Dipartimento Biomedico di Medicina Interna e Specialistica, Università degli Studi di, Palermo, Italy ${ }^{3}$ Dipartimento di Neuroscienze Cliniche, Università degli Studi di, Palermo, Italy

${ }^{4}$ U.O.C. Medicina Clinica

Respiratoria, Dipartimento

Biomedico di Medicina Interna

e Specialistica, Università degli

Studi di, Palermo, Italy

Correspondence to: Dr Carmelo Buttà, U.O.C. Medicina Vascolare, Dipartimento Biomedico di Medicina Interna e Specialistica, Università degli Studi di Palermo, Piazza delle Cliniche $n^{\circ}$ 2, 90127 Palermo, Italy Tel.: + 390916552150 Fax: + 390916552150 Email: carmelob147@tiscali.it

\section{SUMMARY}

Background: This study aimed to evaluate the use of QT intervals, their diagnostic predictive value in patients with syncope and their relationship with syncope severity. Methods: One hundred and forty nine patients with a diagnosis of syncope were admitted to Internal Medicine departments at the University of Palermo, Italy, between 2006 and 2012, and 140 control subjects hospitalised for other causes were enrolled. QT maximum, QT minimum, QTpeak, QT corrected, QT dispersion and Tpeak-to-Tend interval were compared between two groups. The paper medical records were used for scoring with San Francisco Syncope Rule (SFSR), Evaluation of Guidelines in SYncope Study (EGSYS) score and Osservatorio Epidemiologico sulla Sincope nel Lazio (OESIL) risk score. Results: Mean QTC $(p<0.0005)$, mean QTmax $(p<0.0005)$, mean QTdisp $(p<0.0005)$, mean QTpeak $(p=0.005)$ and mean TpTe $(p=0.018)$ were significantly longer in patients with syncope compared with control subjects. A QTc $>424.8 \mathrm{~ms}$ (sensibility: 81.88 - specificity: 57.86 ) showed the greatest predictive value for diagnosis of syncope. On the EGSYS score and on the OESIL score, QTC was significantly prolonged in high-risk patients compared with low-risk patients. On the San Francisco Syncope Rule, QTc and QTdisp were significantly prolonged in high-risk patients compared with low-risk patients. Conclusion: Mean QTc, mean QTdisp, mean TpTe, mean QTmax and mean QTpeak were significantly longer in patients with syncope compared with control subjects. Furthermore, prolonged QTc and QTdisp were associated with major severe syncope according to San Francisco Syncope Rule, EGSYS and OESIL risk scores.

\section{What's known}

A study found that a QTC $\geq 500$ msec was a predictor of increased mortality in emergency department patients with syncope. An increased QTdisp is found in various cardiac diseases in several studies and it is associated with a major risk of torsades de pointes tachycardia. A prolonged TpTe interval is associated with increased risk of mortality in long-QT syndromes, in hypertrophic cardiomyopathy and in coronary artery diseases.

\section{What's new}

In our study, mean QTc, mean QTdisp, mean TpTe, mean QTmax, and mean QTpeak were significantly longer in patients with syncope compared to control subjects. Furthermore, prolonged QTC and QTdisp were associated to major severe syncope according to San Francisco Syncope Rule, EGSYS and OESIL risk scores.
Disclosure

None.

\section{Introduction}

Although syncope is usually considered a benign symptom, it is occasionally associated with significant morbidity and mortality, which seems to be related to the severity of the underlying disease rather than to syncope per se $(1,2)$. Some risk scores evaluated the prognosis of patients affected by syncope; they are the San Francisco Syncope Rule (SFSR) (3), the EGSYS (Evaluation of Guidelines in SYncope Study) score (4) and the OESIL (Osservatorio Epidemiologico sulla Sincope nel Lazio) risk score (5). Generally, in patients with syncope, the major risk factors for sudden cardiac death and overall mortality are: structural heart disease (4-7) and primary electrical disease $(8,9)$. Structural heart disease is a very common predisposing condition for QT corrected (QTc) prolongation (10-12); a long
QTc interval on the surface electrocardiogram (ECG) is often associated with precipitation of torsade de pointes, a polymorphic ventricular tachycardia that may cause syncope, ventricular fibrillation and sudden death not infrequently $(1,11)$.

In order to understand accurately the link between the dispersion of ventricular recovery times, arrhythmias, heart diseases and mortality, in recent years new measurements of QT intervals have been proposed and used. These are the QT dispersion (QTdisp), an index of the spatial dispersion of the ventricular recovery times (13) and the Tpeak-toTend $(\mathrm{TpTe})$ interval, that is a measure of transmural dispersion of repolarisation in the left ventricle (14).

Despite the availability of advanced diagnostic tests, a significant proportion of patients with syncope remain often misdiagnosed and undiagnosed 
especially for the periodic and unpredictable nature of events $(15,16)$.

In our study, we used QT intervals in order to evaluate their predictive value in hospitalised patients towards syncope diagnosis and their relationship with syncope severity.

\section{Materials and methods}

The patients enrolled in our study had a diagnosis of syncope and were admitted to the Operating Units of 'Circulatory Physiopathology', 'Vascular Medicine' and 'Internal Medicine and Cardioangiology' at the University of Palermo, Italy, between January 2006 and June 2012. Anamnesis, laboratory data and demographic, clinical and therapeutic characteristics were collected by reviewing the paper medical records. Syncope was defined as a T-LOC because of transient global cerebral hypoperfusion, characterised by rapid onset, short duration and complete spontaneous recovery (1). Every non-syncope loss of consciousness such as seizure, vertigo, hypoglycaemia, dizziness, head trauma, coma, shock, cataplexy, drop attacks and other states of altered mental status were excluded. The control subjects were patients hospitalised for other causes, in the same period, at the same Operating Units.

On the basis of anamnesis and clinical data, syncope was classified in five groups: reflex (in response to a trigger), orthostatic (secondary to an abnormal decrease in systolic blood pressure upon standing), cardiac or cardiovascular (secondary to arrhythmias or structural cardiovascular diseases), multifactor (if more causes were involved) and unexplained (unmistakable cause).

The ECG, anamnesis, clinical and laboratory data were used for scoring with SFSR, EGSYS score and OESIL risk score. According to the SFSR (3), highrisk patients are those having at least one of the following risk factors: a history of congestive heart failure, haematocrit lower than 30\%, abnormal ECG, a complaint of shortness of breath and systolic blood pressure values lower than $90 \mathrm{~mm} \mathrm{Hg}$. The point score of EGSYS (4) is found as the sum of the following risk factors: palpitations 4, abnormal ECG/Cardiopathy 3, effort syncope 3, syncope in supine position 2, neurovegetative prodromes -1 , precipitating and predisposing factors -1 . A score greater than 2 implies an increased risk of cardiac syncope. According to the OESIL risk score (5), subjects with at least two of these risk factors were assumed to be at intermediate or high risk: abnormal ECG, a previous history of cardiovascular diseases, absence of prodromal symptoms and age over 65 years.
All 12-lead ECGs were performed at $25 \mathrm{~mm} / \mathrm{s}$ paper speed and $10 \mathrm{~mm} / \mathrm{mV}$ amplitude. QT intervals were measured manually on all possible leads by three clinicians blinded to all clinical details in an independent manner. QT interval was defined as the interval from the onset of the QRS complex to the end of the $\mathrm{T}$ wave, which was defined as its return to the T-P baseline. If $\mathrm{U}$ wave was present, the QT interval was measured to the nadir of the curve between the $\mathrm{T}$ and $\mathrm{U}$ waves. If the $\mathrm{T}$-wave amplitude was $<1.5 \mathrm{~mm}$ in a particular lead, that lead was excluded from the analysis. No subject had less than nine measurable leads. QT minimum (QTmin) and QT maximum (QTmax) were defined, respectively, as the minimum and the maximum QT interval measured in all 12 leads. QTpeak was defined as the interval from the onset of the QRS complex to the apex of $\mathrm{T}$ wave on the lead where the QTmax was measured. Measurements of QTmin, QTmax and QTpeak intervals were done in three consecutive cardiac cycles and average values were obtained. QTdisp was defined as the difference between QTmax and QTmin. TpTe was defined as the difference between QTmax and QTpeak. QTc was the QTmax corrected with the Bazett formula to compensate for its known dependence on heart rate: $\mathrm{QT} c=\mathrm{QT} / \sqrt{ } \mathrm{RR}$. QTdisp was not corrected for heart rate because its dependence on heart rate has never been demonstrated (13).

\section{Statistical analysis}

Statistical analysis of quantitative and qualitative data, including descriptive statistics, was performed for all items. Continuous data are expressed as mean $\pm S D$, unless otherwise specified. Baseline differences between groups were assessed by the $\chi^{2}$ test or Fisher's exact test, as needed for categorical variables, and by the independent Student $t$-test for continuous parameters. The univariate analysis of variance (ANOVA) was performed for parametric variables, and post hoc analysis with the Bonferroni test was used to determine whether there were pair-wise differences. Linear regression analysis examined the correlation between patient characteristics (independent variables) and QT measurements (dependent variable) in simple and multiple regression models. Regression coefficients $(B)$ and their 95\% confidence intervals (CIs) were adjusted for sex, previous stroke, coronary artery disease (CAD), diabetes mellitus and beta-blocker use. To assess the predictive role towards syncope diagnosis of different cut-off values of QT measurements, a receiver operating characteristic (ROC) curve with calculations of area under the curve and 95\% CIs was constructed and sensitivity and specificity values were calculated. 
Data were analysed by the Epi Info software (version 6.0, Center for Disease Control and Prevention, Atlanta, GA) and Spss Software (version 14.0, SPSS Inc, Chicago, IL). All p-values were two-sided and p-values less than 0.05 were considered statistically significant.

\section{Results}

We selected 179 consecutive patients admitted to the Operating Units of 'Circulatory Physiopathology', 'Vascular Medicine' and 'Internal Medicine and Cardioangiology' between January 2006 and June 2012. Six patients were excluded because of missing data or poor quality ECG. Patients with atrial fibrillation $(n=13)$, bundle branch block $(n=3)$ and implantable devices $(n=8)$ were also excluded. Medical records of the remaining 149 patients were further reviewed to extract data. Twenty-eight patients had reflex syncope $(18.79 \%), 23$ orthostatic syncope (15.44\%), 13 cardiac syncope (8.72\%), 14 multifactor syncope $(9.40 \%)$ and 71 unexplained syncope (47.65\%). Demographic, clinical and therapeutic characteristics of cases and controls are presented in Table 1. In patients with syncope, there were more subjects of the female sex, with diabetes mellitus and previous stroke; in controls, in contrast, there were more subjects taking beta-blockers and with CAD.

On the SFSR, 67 patients obtained score 0 $(44.97 \%), 57$ had score 1 (38.26\%), 22 score 2 $(14.77 \%)$ and three score $3(2.01 \%)$; no patients had score 4 or 5 ; so there were $82(55.03 \%)$ high-risk

Table 1 Demographic, pathological and therapeutic characteristics of cases and controls

\begin{tabular}{|c|c|c|c|}
\hline Variables & Cases & Controls & $\mathbf{p}^{*}$ \\
\hline Patients & 149 & 140 & \\
\hline Age $( \pm S D)$ & $67(17)$ & $65.9(18)$ & $0.593^{\dagger}$ \\
\hline Sex (male/female) & $56 / 93$ & $99 / 41$ & $<0.0005$ \\
\hline Arterial hypertension (\%) & $109(73.2)$ & $93(66.4)$ & 0.213 \\
\hline Diabetes mellitus & $58(38.9)$ & $25(17.9)$ & $<0.0005$ \\
\hline Coronary artery disease & $33(22.1)$ & $53(37.9)$ & 0.004 \\
\hline Previous stroke & $15(10.1)$ & $3(2.1)$ & 0.005 \\
\hline Chronic kidney disease & $7(4.7)$ & $2(1.4)$ & 0.110 \\
\hline $\begin{array}{l}\text { Chronic obstructive } \\
\text { pulmonary disease }\end{array}$ & $4(2.7)$ & $8(5.7)$ & 0.197 \\
\hline Beta-blockers & $25(16.8)$ & $57(40.7)$ & $<0.0005$ \\
\hline $\begin{array}{l}\text { Antiarrhythmics } \\
\text { (IC and III classes) }\end{array}$ & $5(3.4)$ & $2(1.4)$ & 0.448 \\
\hline Psycholeptics & $7(4.7)$ & $5(3.6)$ & 0.631 \\
\hline \multicolumn{4}{|c|}{$\begin{array}{l}\text { SD, standard deviation. *Pearson's } \chi^{2} \text { test or Fisher's exact } \\
\text { test, as needed; †'Independent student t-test. } \\
\text { Statistically significant }(p<0.05 \text { ) is shown in bold. }\end{array}$} \\
\hline
\end{tabular}

patients. On the EGSYS score, 23 patients had score -1 (15.44\%), 37 had score $0(24.83 \%)$, one patient score $1(0.67 \%), 18$ patients score $2(12.08 \%), 66$ score $3(44.30 \%)$, one patient score $5(0.67 \%)$, two patients score $6(1.34 \%)$ and one patient score 7 $(0.67 \%)$, no patients had score $-2,4$ or $>7$. So on EGSYS, there were 70 patients $(46.98 \%)$ with an increased risk of cardiac syncope. On OESIL risk score, 31 patients had score 0 (20.81\%), 39 had score 1 (26.17\%), 30 score 2 (20.13\%), 30 score 3 $(20.13 \%)$ and 19 score $4(12.75 \%)$; so there were 79 (53.02\%) patients with intermediate or high risk.

Mean QTmax, mean QTmin, mean QTpeak, mean QTc, mean QTdisp and mean TpTe were significantly longer in patients with syncope compared with control subjects at univariate ANOVA analysis; these variables, except QTmin, were prolonged in patients with syncope even after adjustment for sex, diabetes mellitus, CAD, previous stroke and betablockers (see Table 2).

According to the SFSR, patients with score 1 and 2 had longer mean QTc compared with patients with score 0 and patients with score 1 had longer mean QTdisp compared with patients with score 0 . On the EGSYS score, patients with score 3 had longer mean QTc compared with patients with score 0 . On the OESIL score, patients with score 2 and 3 had longer mean QTc than the patients with score 0 .

Concerning the demographic, clinical and therapeutic variables analysed on multivariable logistic regression model, CAD was significantly associated with longer mean QTmax, mean QTpeak, mean QTmin (see Table 3) and mean QTdisp (see Table 4), and beta-blockers were associated with shorter mean QTdisp and mean TpTe (see Table 4).

In order to evaluate the diagnostic power of QT measurements in the syncope setting, we assessed the predictive role of different cut-off values for syncope diagnosis at ROC curve analysis: QTc > $424.8 \mathrm{~ms}$ (sensibility: 81.88 - specificity: 57.86), QTdisp $>50 \mathrm{~ms}$ (sensibility: 48.99 - specificity: 81.43), QTmax > $410 \mathrm{~ms}$ (sensibility: 46.31 - specificity: 83.57), TpTe $>100$ ms (sensibility: 31.54 - specificity: 89.29) and QTpeak > $330 \mathrm{~ms}$ (sensibility: 30.87 specificity: 82.86 ), which showed a significant predictive value with an area under the ROC curve of $74.7 \%, 70.8 \%, 65.4 \%, 59.6 \%$ and $59.2 \%$, respectively (see Figure 1).

\section{Discussion}

To the best of our knowledge, this is the first study that evaluates the diagnostic power of QT intervals in patients with syncope. In our study, mean QTc, mean QTdisp, mean TpTe, mean QTmax and mean 
Table 2 QT measurements and heart rate (mean values \pm SD)

\begin{tabular}{lllcc}
\hline Measurements & Cases & Controls & $\mathbf{p}^{*}$ & $\mathbf{p}^{\boldsymbol{*}}$ \\
\hline QTmax (ms) & $413.7(65.3)$ & $385.3(33.8)$ & $<\mathbf{0 . 0 0 0 5}$ & $<\mathbf{0 . 0 0 0 5}$ \\
QTmin (ms) & $354.8(51.1)$ & $343.6(30.7)$ & $\mathbf{0 . 0 2 6}$ & 0.094 \\
QTpeak (ms) & $319.1(56.2)$ & $301.5(33.2)$ & $\mathbf{0 . 0 0 1}$ & $\mathbf{0 . 0 0 5}$ \\
Heart rate (bpm) & $76.6(16.9)$ & $74.3(12.9)$ & 0.202 & 0.592 \\
QTc (ms) & $459.4(47.6)$ & $425.2(34.3)$ & $<0.0005$ & $<0.0005$ \\
QTdisp (ms) & $58.8(30.8)$ & $41.7(21.3)$ & $\mathbf{0 . 0 0 0 5}$ & $\mathbf{0 . 0 0 0 5}$ \\
TpTe (ms) & $94.5(30.2)$ & $83.8(22.3)$ & $\mathbf{0 . 0 0 1}$ & $\mathbf{0 . 0 1 8}$ \\
\hline
\end{tabular}

*Univariate ANova; ${ }^{\dagger}$ ANova analysis adjusted for sex, diabetes mellitus, coronary artery disease, previous stroke and beta-blockers.

Statistically significant $(p<0.05)$ is shown in bold.

Table 3 Multiple regression analysis of QTmax, QTpeak, and Qtmin in patients with syncope

\begin{tabular}{|c|c|c|c|}
\hline Regression models & Coefficient (B) & $95 \%$ confidence interval & p \\
\hline \multicolumn{4}{|l|}{ QTmax } \\
\hline QTmax (univariate analysis) & 28.334 & $16.166-40.502$ & $<0.0005$ \\
\hline QTmax (multivariate analysis) & 31.072 & $14.668-47.477$ & $<0.0005$ \\
\hline Sex & -6.931 & $-19.386-5.525$ & 0.274 \\
\hline Previous stroke & -15.165 & $-39.787-9.456$ & 0.226 \\
\hline Coronary artery disease & 32.424 & $17.584-47.263$ & $<0.0005$ \\
\hline Diabetes mellitus & -3.669 & $-17.212-9.875$ & 0.594 \\
\hline Beta-blockers & 1.470 & $-13.318-16.259$ & 0.845 \\
\hline \multicolumn{4}{|l|}{ QTpeak } \\
\hline QTpeak (univariate analysis) & 17.661 & $6.873-28.449$ & 0.001 \\
\hline QTpeak (multivariate analysis) & 20.538 & $6.136-34.939$ & 0.005 \\
\hline Sex & -10.727 & $-21.661-0.208$ & 0.054 \\
\hline Previous stroke & -12.833 & $-34.449-8.782$ & 0.244 \\
\hline Coronary artery disease & 27.794 & $14.766-40.822$ & $<0.0005$ \\
\hline Diabetes mellitus & 1.596 & $-10.294-13.486$ & 0.792 \\
\hline Beta-blockers & 10.542 & $-2.442-23.525$ & 0.111 \\
\hline \multicolumn{4}{|l|}{ QTmin } \\
\hline QTmin (univariate analysis) & 11.189 & $1.344-21.035$ & 0.026 \\
\hline QTmin (multivariate analysis) & 11.550 & $-1.980-25.080$ & 0.094 \\
\hline Sex & -7.732 & $-18.005-2.540$ & 0.140 \\
\hline Previous stroke & -12.236 & $-32.543-8.072$ & 0.237 \\
\hline Coronary artery disease & 17.434 & 5.194-29.673 & 0.005 \\
\hline Diabetes mellitus & -6.377 & $-17.548-4.793$ & 0.262 \\
\hline Beta-blockers & 9.917 & $-2.281-22.115$ & 0.111 \\
\hline
\end{tabular}

QTpeak were significantly longer in patients with syncope compared with control subjects. Among these variables, a QTc $>424.8 \mathrm{~ms}$ showed the greatest predictive value for diagnosis of syncope.

Among the demographic, clinical and therapeutic variables analysed at multivariable logistic regression model, CAD was associated with prolonged mean QTmax, mean QTpeak, mean QTmin and mean QTdisp.

A study of Aggarwal et al. (17) showed a link between CAD and prolonged QTc in patients with syncope. In our study, a link between CAD and prolonged QTc was not found even though the patients with CAD had a prolonged mean QTmax. This discordance was probably because of the fact that the patients with CAD took more beta-blockers than non-ischaemic subjects and consequently had lower heart rate. Therefore, after QTmax corrected for heart rate, QTc was not significantly different in ischaemic and non-ischaemic patients. Although some authors did not find significant differences between patients with chronic CAD and normal subjects 
Table 4 Regression analysis of QTc, QTdisp and TpTe in patients with syncope

\begin{tabular}{|c|c|c|c|}
\hline Regression models & Coefficient (B) & $95 \%$ confidence interval & $\mathrm{p}$ \\
\hline \multicolumn{4}{|l|}{ QTC } \\
\hline QTc (univariate analysis) & 34.134 & $24.464-43.805$ & $<0.0005$ \\
\hline QTc (multivariate analysis) & 27.597 & $14.156-41.038$ & $<0.0005$ \\
\hline Sex & -5.859 & $-16.064-4.347$ & 0.259 \\
\hline Previous stroke & 8.821 & $-11.353-28.995$ & 0.390 \\
\hline Coronary artery disease & 6.654 & $-5.506-18.813$ & 0.282 \\
\hline Diabetes mellitus & 5.089 & $-6.008-16.186$ & 0.367 \\
\hline Beta-blockers & -8.771 & $-20.889-3.346$ & 0.155 \\
\hline \multicolumn{4}{|l|}{ QTdisp } \\
\hline QTdisp (univariate analysis) & 17.145 & 10.964-23.326 & $<0.0005$ \\
\hline QTdisp (multivariate analysis) & 19.522 & $11.049-27.995$ & $<0.0005$ \\
\hline Sex & 0.802 & $-5.631-7.235$ & 0.806 \\
\hline Previous stroke & -2.930 & $-15.647-9.788$ & 0.651 \\
\hline Coronary artery disease & 14.990 & 7.325-22.655 & $<0.0005$ \\
\hline Diabetes mellitus & 2.709 & $-4.287-9.704$ & 0.447 \\
\hline Beta-blockers & -8.447 & -16.085 to -0.808 & 0.030 \\
\hline \multicolumn{4}{|l|}{ TpTe } \\
\hline TpTe (univariate analysis) & 10.673 & $4.497-16.849$ & 0.001 \\
\hline TpTe (multivariate analysis) & 10.535 & 1.841-19.229 & 0.018 \\
\hline Sex & 3.796 & $-2.805-10.397$ & 0.259 \\
\hline Previous stroke & -2.332 & $-15.381-10.717$ & 0.725 \\
\hline Coronary artery disease & 4.630 & $-3.235-12.495$ & 0.248 \\
\hline Diabetes mellitus & -5.265 & $-12.442-1.913$ & 0.150 \\
\hline Beta-blockers & -9.071 & -16.909 to -1.234 & 0.023 \\
\hline
\end{tabular}

Statistically significant $(p<0.05)$ is shown in bold.

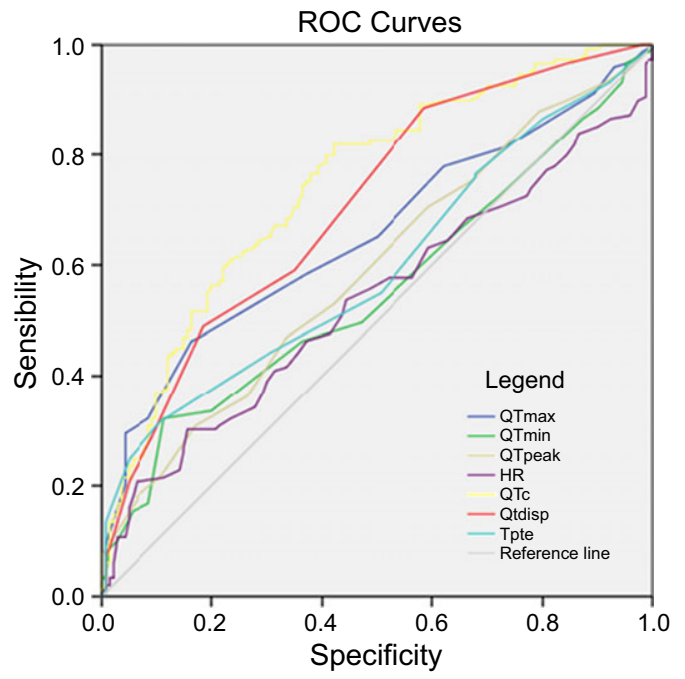

Figure 1 Predictive role (sensibility and specificity) of electrocardiographic indexes (QT max, QT min, QT peak, QT dispersion, QTc, TpTe) towards syncope diagnosis

$(18,19)$, QTdisp is generally longer in the chronic phase of myocardial infarction (13). It was interesting that, in our study, mean QTmax, mean QTpeak and mean QTdisp were longer in patients with syncope than controls, although the control subjects more frequently had CAD compared with patients with syncope. A possible reason for this observation is that the patients with syncope might have more severe structural non-ischaemic cardiopathy than controls; these cardiopathies could be myocardial fibrosis, left ventricular hypertrophy or cardiac autonomic neuropathy (12). So, QT intervals could depend on severity of underlying structural cardiopathy rather than $\mathrm{CAD}$ and, therefore, they could represent a marker of this severity.

Usually, women have longer QTc $(14,20)$ and shorter QTdisp (21) and TpTe (22) than men. In our study, the differences in ventricular repolarisation measurements between cases and controls seem not to depend on sex differences between two groups owing to the fact that on multivariable logistic regression model, there are no significant differences between women and men. The marked sex differences in ventricular repolarisation could be explained by differences in circulating sex hormones in women and men $(14,23)$. Since the patients in our study were mostly elderly people, the differences in sex hormones were less marked between women and men and, therefore, the ven- 
tricular repolarisation measurements did not differ significantly.

Some studies found QTc and QTdisp significantly longer in diabetic patients compared with non-diabetic patients (24-26). These differences seem to depend more on the cardiac autonomic neuropathy, secondary to diabetes mellitus than to diabetes mellitus per se $(27,28)$. In our study, the differences in ventricular repolarisation measurements in diabetic and non-diabetic patients were not significant.

A study by Seftchick et al. (10) found that previous stroke is a common predisposing condition for QTc prolongation. Our study does not confirm this finding, possibly because the rate of patients with previous stroke was low.

Some studies found a significant reduction in QTdisp and TpTe in patients with arterial hypertension, long-QT syndrome and heart failure taking beta-blockers (29-31), supporting the theory that beta-blocker therapy reduces dispersion of repolarisation and protects patients from the onset of ventricular arrhythmias. Our study confirmed these findings showing that beta-blockers were significantly associated with shorter mean QTdisp and mean TpTe on multivariable logistic regression (see Table 4).

A study by Aggarwal et al. (17) evaluated the prognosis of QT interval in emergency department patients with syncope; they found that a QTc $\geq 500 \mathrm{~ms}$ was a predictor of increased mortality among those patients. In our study, we related QT intervals of patients with syncope with different scores according to SFSR, EGSYS and OESIL scores.

The SFSR (3) is a study that evaluated the onset of serious outcomes in patients with syncope within 7 days of their emergency department visit; therefore, it evaluated the short-term prognosis. The presence of a score $\geq 1$ distinguished patients at high risk of serious outcomes with a sensitivity of $96 \%$ and a specificity of $62 \%$. Our study showed a significantly longer mean QTc in patients with score 1 and 2 than the patients with score 0 and a significantly longer mean QTdisp in patients with score 1 than in those with score 0 . The lack of statistical significance for higher scores is probably because of the reduced number of these subgroups. Therefore, in our study a prolonged QTc and a prolonged QTdisp could be additional markers of worse short-term prognosis.

Evaluation of Guidelines in SYncope Study is a validated score used to identify those patients likely to have a cardiac syncope (4) and to predict the long-term mortality (follow up of 21-24 months) of patients with syncope (32). Patients at high risk had a score $\geq 3$. In our study, patients with score 3 had a longer mean QTc than those with score 0. OESIL is a risk stratification system used to identify independent predictors of total mortality within the first 12 months after the initial evaluation of emergency department patients with syncope (5). Subjects with a score of 2 or higher were assumed to be at intermediate or high risk. In our study, patients with score 2 and 3 had a longer mean QTc than patients with score 0 . The lack of statistical significance for higher scores in both EGSYS and OESIL scores is probably because of the reduced number of these subgroups. Therefore, according to our study it is possible to affirm that a prolonged QTc could be a predictive marker of a worse long-term prognosis and of a diagnosis of cardiac syncope. Because $8.72 \%$ of our patients had a diagnosis of cardiac syncope and $46.98 \%$ had a likely cardiac cause according to the EGSYS score, it is possible that a high rate of cardiac syncope was misdiagnosed or undiagnosed in our study. Therefore, QTc could be proposed to reduce the diagnostic errors in these patients.

\section{Study limitations}

Our study has certain limitations. A larger sample would certainly increase the statistical power of the study. The retrospective design may cause missing data regarding the diagnosis or the aetiology of syncope. Manual measurements of intervals without the support of any technology that could ensure a more precise measurement may also be an aspect to be taken into account.

\section{Conclusions}

In conclusion, our study demonstrated that, in a population of patients admitted to internal medicine departments, QTc > $424.8 \mathrm{~ms}$ (sensibility: 81.88 specificity: 57.86), QTdisp > $50 \mathrm{~ms}$ (sensibility: 48.99 - specificity: 81.43), QTmax > $410 \mathrm{~ms}$ (sensibility: 46.31 - specificity: 83.57 ), TpTe $>100 \mathrm{~ms}$ (sensibility: 31.54 - specificity: 89.29 ) and QTpeak > $330 \mathrm{~ms}$ (sensibility: 30.87 - specificity: 82.86 ) had a predictive value for diagnosis of syncope; therefore, QT measurements could help the clinician to diagnose syncope in patients who have an unclear anamnesis.

Furthermore, prolonged QTc and QTdisp were associated with high risk of serious outcomes at SFSR and prolonged QTc was associated with intermediate and high risk at EGSYS and OESIL risk scores; therefore, QTc and QTdisp could be considered as possible independent markers of syncope severity beyond the current understanding of their arrhythmic potential.

Further studies are required to elucidate the validity of our clinical findings. 


\section{References}

1 Moya A, Sutton R, Ammirati F et al. Guidelines for the diagnosis and management of syncope (version 2009). Eur Heart J 2009; 30(21): 2631-71.

2 Quinn J, McDermott D, Stiell I, Kohn M, Wells G. Prospective validation of the San Francisco Syncope Rule to predict patients with serious outcomes. Ann Emerg Med 2006; 47(5): 448-54.

3 Quinn JV, Stiell IG, McDermott DA, Sellers KL, Kohn MA, Wells GA. Derivation of the San Francisco Syncope Rule to predict patients with short-term serious outcomes. Ann Emerg Med 2004; 43: 224-32.

4 Del Rosso A, Ungar A, Maggi R et al. Clinical predictors of cardiac syncope at initial evaluation in patients referred urgently to a general hospital: the EGSYS score. Heart 2008; 94: 1620-6.

5 Colivicchi F, Ammirati F, Melina D, Guido V, Imperoli G, Santini M. Development and prospective validation of risk stratification system for patients with syncope in the emergency department: the OESIL risk score. Eur Heart J 2003; 24: 811-9.

6 Martin TP, Hanusa BH, Kapoor WN. Risk stratification of patients with syncope. Ann Emerg Med 1997; 29: 459-66.

7 Olshansky B, Poole JE, Johnson G et al. Syncope predicts the outcome of cardiomyopathy patients: analysis of the SCD-HeFT study. J Am Coll Cardiol 2008; 51: 1277-82.

8 Wehrens XH, Vos MA, Doevendans PA, Wellens HJ. Novel insights in the congenital long QT syndrome. Ann Intern Med 2002; 137: 981-92.

9 Antzelevitch C, Brugada P, Borggrefe $\mathrm{M}$ et al. Brugada syndrome: report of the Second Consensus Conference. Circulation 2005; 111: 659-70.

10 Seftchick MW, Adler PH, Hsieh M et al. The prevalence and factors associated with QTc prolongation among emergency department patients. Ann Emerg Med 2009; 54: 763-8.

11 Kallergis EM, Goudis CA, Simantirakis EN, Kochiadakis GE, Vardas PE. Mechanisms, risk factors, and management of acquired long QT syndrome: a comprehensive review. ScientificWorldJournal 2012; 2012: 212178 .
12 Ouali S, Ben Salem H, Gribaa R et al. The QT interval: standardization, limits and interpretation. Ann Cardiol Angeiol (Paris) 2012; 61(1): 42-8.

13 Malik M, Batchvarov VN. Measurement, interpretation and clinical potential of QT dispersion. J Am Coll Cardiol 2000; 36: 1749-66.

14 Panikkath R, Reinier K, Uy-Evanado A et al. Prolonged Tpeak-to-Tend interval on the resting ECG is associated with increate risk of sudden cardiac death. Circ Arrhythm Electrophysiol 2011; 4: 441-7.

15 Palaniswamy C, Aronow WS, Agrawal N, Balasubramaniyam N, Lakshmanadoss U. Syncope: approaches to diagnosis and management $A m \mathrm{~J}$ Ther 2012. [Epub ahead of print].

16 Krahn AD, Klein GJ, Yee R, Skanes AC. The use of monitoring strategies in patients with unexplained syncope - role of the external and implantable loop recorder. Clin Auton Res 2004; 14(1): 55-61.

17 Aggarwal A, Sherazi S, Levitan B et al. Corrected QT interval as a predictor of mortality in elderly patients with syncope. Cardiol J 2011; 18(4): 395400.

18 Ashikaga T, Nishizaki M, Arita M et al. Effect of dipyridamole on QT dispersion in vasospastic angina pectoris. Am J Cardiol 1999; 84: 807-10.

19 Sporton SC, Taggart P, Sutton PM, Walker JM, Hardman SM. Acute ischemia: a dynamic influence on QT dispersion. Lancet 1997; 349: 306-9.

20 Pham TV, Rosen MR. Sex, hormones, and repolarization. Cardiovasc Res 2002; 53: 740-51.

21 Tran H, White CM, Chow MS, Kluger J. An evaluation of the impact of gender and age on QT dispersion in healthy subjects. Ann Noninvasive Electrocardiol 2001; 6(2): 129-33.

22 Nakagawa $M$, Takahashi $\mathrm{N}$, Watanabe $\mathrm{M}$ et al. Gender differences in ventricular repolarization: terminal $\mathrm{T}$ wave interval was shorter in women than in men. Pacing Clin Electrophysiol 2003; 26: 59-64.

23 Cheng J. Evidences of the gender-related differences in cardiac repolarization and the underlying mechanisms in different animal species and human. Fundam Clin Pharmacol 2006; 20(1): 1-8.
24 Clemente D, Pereira T, Ribeiro S. Ventricular repolarization in diabetic patients: characterization and clinical implications. Arq Bras Cardiol 2012; 99(5): 1015-22.

25 Arildsen H, May O, Christiansen EH, Damsgaard EM. Increased QT dispersion in patients with insulin-dependent diabetes mellitus. Int J Cardiol 1999; 71(3): 235-42.

26 Jermendy G, Koltai MZ, Pogatsa G. QT interval prolongation in type 2 (non-insulin-dependent) diabetic patients with cardiac autonomic neuropathy. Acta Diabetol Lat 1990; 27(4): 295-301.

27 Takahashi N, Nakagawa M, Saikawa T et al. Regulation of QT indices mediated by autonomic nervous function in patients with type 2 diabetes. Int $\mathrm{J}$ Cardiol 2004; 96(3): 375-9.

28 Matel D, Chiochin AD, Stratone A. Utility of QTc interval for the diagnosis of cardiac autonomic neuropathy in type 2 diabetes mellitus. Rev Med Chir Soc Med Nat Iasi 2010; 114(1): 282-6.

29 Galetta F, Franzoni F, Magagna A et al. Effect of nebivolol on QT dispersion in hypertensive patients with left ventricular hypertrophy. Biomed Pharmacother 2005; 59(1-2): 15-9.

30 Aygul N, Ozdemir K, Duzenli MA, Aygul MU. The comparative effects of long-term carvedilol versus bisoprolol therapy on QT dispersion in patients with chronic heart failure. Cardiology 2009; 112(3): 168-73.

31 Viitasalo M, Oikarinen L, Swan $\mathrm{H}$ et al. Effect of beta-blocker therapy on ventricular repolarization documented by 24-h electrocardiography in patients with type 1 long-QT syndrome. J Am Coll Cardiol 2006; 48(4): 747-53.

32 Shimizu M, Ino $\mathrm{H}$, Okeie $\mathrm{K}$ et al. T-peak to T-end interval may be a better predictor of high-risk patients with hypertrophic cardiomyopathy associated with a cardiac troponin I mutation than QT dispersion. Clin Cardiol 2002; 25: 335-9.

Paper received November 2013, accepted December 2013 